\title{
Resultados del tratamiento quirúrgico del cáncer del tercio superior del recto y de colon sigmoides: Análisis comparativo*
}

\author{
Drs. GUILlERMO BANNURA C. ${ }^{1}$, MIGUEL A. CUMSIllE G. ${ }^{2}$, ALEJANDRO BARRERA E. ${ }^{1}$, \\ JAIME CONTRERAS P. ${ }^{1}$, CARLOS MELO L. ${ }^{1}$, DANIEL SOTO C. ${ }^{1}$
}

1 Servicio y Departamento de Cirugía, Hospital Clínico San Borja Arriarán. Campus Centro.

2 Escuela de Salud Pública.

Facultad de Medicina. Universidad de Chile. Santiago, Chile.

\section{Abstract \\ Comparative results of the surgical treatment of tumors located in the upper third of the rectum and sigmoid colon}

Background: Tumors located in the upper third of the rectum can be treated in the same way as sigmoid tumors. This means that mesorrectum excision and neoadjuvant therapies are not necessary. Aim: To compare the results in terms of relapse and survival of elective surgery for sigmoid and upper third rectal tumors. Material and Methods: Retrospective analysis of patients operated for an upper third rectal or sigmoid cancer that were prospectively included in a database and had a minimum follow up of 18 months. Clinical and pathological variables and staging using TNM classification were considered as covariates in the analysis. Results: Fifty patients with a tumor of the upper third of the rectum, aged 30 to 84 years (24 females), with a follow up ranging from 12 to 206 months, and 107 patients with a sigmoid tumor, aged 22 to 91 years (62 females), with a follow up ranging from 13 to 204 months, were analyzed. Seven patients with rectal tumors (14\%) and 12 patients with sigmoid tumors (11\%), died during follow up $(\mathrm{p}=\mathrm{NS})$. Ten years actuarial overall survival was $85 \%$ for both groups. Conclusions: No differences in survival or relapse were observed in this group of patients with tumors located in the upper third of the rectum or sigmoid colon. Therefore both types of tumors can be treated in the same way.

Key words: Sigmoid cancer, rectal cancer, survival.

\section{Resumen}

Introducción: Se ha sugerido que los pacientes con un tumor del tercio superior del recto (RS) podrían tratarse en forma similar que los portadores de un cáncer de sigmoides (CS). Ello implica no realizar la resección total del mesorrecto ni someterlos a terapias neoadyuvantes. El objetivo de este estudio es comparar los resultados del tratamiento quirúrgico electivo de ambos tumores respecto de la recidiva y la sobrevida global. Pacientes y Método: Análisis retrospectivo de las características demográficas de los pacientes intervenidos por un adenocarcinoma de recto superior y de colon sigmoides con intención curativa en forma electiva en forma consecutiva con un seguimiento mínimo de 18 meses. Se considera

*Recibido el 17 de Marzo de 2009 y aceptado para publicación el 17 de Agosto de 2009.

No hay conflictos de interés en este trabajo. No hubo apoyo económico en la creación del trabajo.

Correspondencia: Dr. Guillermo Bannura C.

Las Limas 1622, Santiago, Chile.

E-mail: gbannura@vtr.net 
variables clínicas (edad, género, enfermedades asociadas, antígeno carcinoembrionario (CEA) preoperatorio), histopatológicas (diferenciación y tamaño tumoral, número de ganglios examinados) y la estadificación de ambos grupos según TNM. Resultados: Se compara 50 pacientes portadores de un adenocarcinoma de RS en los estadíos I a III con 107 pacientes intervenidos en el mismo período por un cáncer de CS con intención curativa. El seguimiento promedio de los pacientes con un tumor de RS fue de 88,62 meses (extremos 12-206), y de 71,67 meses en los casos de CS (extremos 13-204) ( $p=0,05)$. Durante este período fallecen por la enfermedad 7 pacientes (14\%) del grupo RS, 1 por recidiva locorregional y 6 por recidiva a distancia, con un rango entre 12 y 78 meses. En el grupo CS fallecen 12 pacientes $(11,2 \%), 3(2,8 \%)$ con recidiva locorregional y 9 con recidiva a distancia, entre 24 y 82 meses de seguimiento $(\mathrm{p}=0,24)$. La sobrevida actuarial global a 10 años para ambos grupos fue de $85 \%$. Conclusión: En este estudio no existen diferencias en cuanto a la recidiva global, la recidiva local ni la recidiva a distancia entre ambos grupos, lo que avala la estrategia de tratar en forma similar los tumores de ambas localizaciones, evitando tratamientos neoadyuvantes costosos y con serios efectos adversos.

Palabras clave: Cáncer de recto, cáncer de sigmoides, tratamiento quirúrgico.

\section{Introducción}

En el tratamiento del cáncer colorrectal se ha destacado, cada vez con mayor fuerza, la necesidad de separar los tumores del recto (hasta el promontorio) de las lesiones ubicadas en el colon ${ }^{1}$. La técnica de la resección total del mesorrecto (RTM) luego de radioquimioterapia preoperatoria se considera actualmente como parte del manejo estándar de los tumores ubicados en el recto medio y en el recto inferior ${ }^{2,3}$. La resección anterior es la técnica de elección para los tumores ubicados en sigmoides medio e inferior, habitualmente con una anastomosis a la altura del promontorio ${ }^{4}$. En los tumores de colon en estadío III (y discutible en algunos estadíos II) se considera, además, la quimioterapia postoperatoria en pacientes aptos ${ }^{4}$. Se ha sugerido que los tumores del recto superior (RS) tendrían un comportamiento biológico más parecido a los tumores de colon, y por tanto, susceptibles de un manejo similar a los tumores de colon sigmoides $(\mathrm{CS})^{5}$. En estos casos, la cirugía resectiva radical es la piedra angular del tratamiento, logrando bajos índices de recidiva local y una sobrevida global a 5 años del 70 a $85 \%$ en los pacientes resecados con intención curativa ${ }^{6-8} \cdot ¿$ Debe manejarse el cáncer de $\mathrm{RS}$ en forma similar a un cáncer de CS o debe incluirse en los protocolos de radioquimioterapia neoadyuvante como en los tumores de recto bajo? El objetivo de este estudio es comparar los resultados del tratamiento quirúrgico de los tumores de RS y CS, con especial énfasis en los índices de recidiva y sobrevida a largo plazo.

\section{Material y Método}

Se analiza en forma retrospectiva las características demográficas de los pacientes intervenidos por un adenocarcinoma de recto superior y de colon sigmoides con intención curativa en forma electiva en forma consecutiva. La información se obtuvo de una base de datos computarizada confeccionada en forma prospectiva. Se incluyen los pacientes estadío I a III de la clasificación TNM de 1997 (5 edición $)^{9}$ con un seguimiento potencial mínimo de 18 meses. Se excluyen los pacientes sometidos a radioterapia, los estadíos IV y la mortalidad quirúrgica $(1,3 \%)$. Se compara algunas variables clínicas (edad, género, enfermedades asociadas, antígeno carcinoembrionario (CEA) preoperatorio), histopatológicas (diferenciación y tamaño tumoral, número de ganglios examinados) y la estadificación de ambos grupos según el TNM señalado. La concentración sérica del CEA fue determinada por la técnica de inmuno-enzimoanálisis de micropartículas (MEIA, Abbott Laboratories, Chicago, Il), aceptando el valor de $5 \mathrm{ng} / \mathrm{ml}$ como valor normal máximo. Como cáncer del RS se define toda lesión cuyo límite inferior está ubicada entre los 11 y los $15 \mathrm{~cm}$ del margen anal, medición efectuada con instrumento rígido. Los tumores ubicados por encima del promontorio y hasta la unión entre el colon descendente y el colon sigmoides se catalogaron como cáncer de CS. Se considera cirugía con intención curativa los estadíos I a III sometidos a una resección R0, es decir, sin enfermedad macroscópica residual. La intervención quirúrgica principal en ambas localizaciones fue la resección anterior o la hemicolectomía izquierda por laparotomía, según corresponda. En los tumores T4 fistulizados se efectuó una resección en block del órgano afectado. La resección radical debe sobrepasar el tumor al menos $5 \mathrm{~cm}$ hacia distal sin conización del mesorrecto e incluye la ligadura alta de los vasos mesentéricos inferiores y el vaciamiento linfático estándar (no incluye los linfonodos lumboaórticos). La anastomosis colorrectal grapada queda a nivel del promontorio en los tumores de CS y por sobre la reflexión peritoneal en las lesiones de RS. La QT adyuvante 
consistió en la infusión endovenosa de 5-FU (fluoruracilo) $425 \mathrm{mg} / \mathrm{m}^{2}$ más Leucovorina $20 \mathrm{mg} / \mathrm{m}^{2} \mathrm{los}$ días $1-5$ cada 28 días por 6 ciclos.

Como medida indirecta de la magnitud de la cirugía y la morbilidad asociada se comparó el tiempo de hospitalización promedio y la desviación estándar (DE) en cada grupo. Los resultados intermedios del tratamiento quirúrgico se evaluaron de acuerdo al número de ganglios resecados, como indicador de la radicalidad de la cirugía. Para evaluar los resultados finales se consideró los índices de recidiva global, la recidiva locorregional y la sobrevida actuarial a 10 años. La reaparición del tumor en el lecho quirúrgico se define como recidiva locorregional. La recidiva en sitios alejados (hepática, pulmonar, adenopatías lumboaórticas y carcinomatosis peritoneal) se considera recidiva a distancia y la suma de ambas corresponde a la recidiva global. Para el análisis estadístico de asociación se usó la prueba del chi cuadrado y el test de Wilcoxon, según corresponda. Para estimar las curvas de sobrevida se utilizó el método de KaplanMeier. Para comparar las curvas de sobrevida se usó el test de log-rank. Un $\mathrm{p} \leq 0,05$ se consideró estadísticamente significativo.

\section{Resultados}

Veinte y tres $(31,5 \%)$ de un total de 73 pacientes portadores de un cáncer de RS y $39(26,7 \%)$ de un total de 146 pacientes portadores de un tumor de CS fueron etapificados en estadío IV al momento de la cirugía, los que se excluyen del análisis. De este modo, en el período de 16 años analizado se intervinieron 50 pacientes portadores de un adenocarcinoma de RS en los estadíos I a III, los que se comparan con 107 pacientes intervenidos en el mismo período por un cáncer de CS con intención curativa. El tiempo de hospitalización promedio fue de 10,4 días (DE: 5,23; extremos: 5-38) en RS, y 9,53 (DE: 5,25; extremos 4-37) en CS, sin diferencias estadísticamente significativas $(\mathrm{p}=0,33)$. El hematocrito preoperatorio promedio fue 38,8 (DE: 5,06) en RS y 36,7 (DE: 6,13) en CS, lo que alcanza la significación estadística $(\mathrm{p}=0,04)$. Al dicotomizar la variable hematocrito, el $14 \%$ de los pacientes con un tumor de RS tenía un hematocrito $\leq 35 \%$ versus el $40 \%$ en las lesiones de CS $(p=0,003)$. No hubo diferencias estadísticamente significativas entre ambos grupos en el resto de las variables consideradas y, particularmente, la distribución en los estadíos I a III es homogénea (Tabla 1). Sin embargo, hubo una mayor concentración de tumores perforados o fistulizados (T4) en el grupo CS comparado con el grupo $\mathrm{RS}(\mathrm{p}=0,01)$. El número promedio de ganglios examinados fue en RS 14,08 (DE: $6,86)$ versus 14,35 en CS (DE: 8,13) $(\mathrm{p}=0,20)$. En forma global, el $26 \%$ y el $34,5 \%$ de los pacientes con tumores de RS y de CS, respectivamente, fueron sometidos a quimioterapia adyuvante $(\mathrm{p}=0,54)$. Sin embargo, hubo una mayor proporción de pa-

Tabla 1. Análisis comparativo de los pacientes con tumores de RS y CS

\begin{tabular}{lccc}
\hline Variable & $\begin{array}{c}\text { Recto superior } \\
\mathbf{n = 5 0}\end{array}$ & $\begin{array}{c}\text { Colon sigmoides } \\
\mathbf{n = 1 0 7}\end{array}$ & $\mathbf{p}$ \\
\hline Edad promedio (extremos) & $66,3(30-84)$ & $65,7(22-91)$ & 0,75 \\
Género (mujer/hombre) & $24 / 26$ & $62 / 45$ & 0,24 \\
Enfermedades asociadas (\%) & 56 & 47 & 0,28 \\
Albúmina preoperatoria promedio & 3,95 & 4 & 0,52 \\
CEA preoperatorio promedio (extremos) & $7,5(0,6-60,2)$ & $10,2(0,2-192)$ & 0,51 \\
Tumores indiferenciados (\%) & 4 & 2 & 0,33 \\
Tamaño tumoral (cm) & 5,58 & 5,46 & 0,75 \\
No ganglios examinados & 14,08 & 14,35 & 0,20 \\
Variable T & & & \\
$\quad$ T1-T2 & 14 & 18 & 0,10 \\
T3-T4 & 36 & 89 & \\
TNM (1977) & 12 & & \\
$\quad$ Estadío I & 20 & 16 & 0,30 \\
\multicolumn{1}{|l}{ II } & 18 & 54 & 37 \\
\hline
\end{tabular}

CEA: antígeno carcinoembrionario 
Tabla 2. Análisis comparativo de la recidiva y la sobrevida

\begin{tabular}{lcrc} 
& $\begin{array}{c}\text { Recto superior } \\
\mathbf{n = 5 0}\end{array}$ & $\begin{array}{c}\text { Colon sigmoides } \\
\mathbf{n}=\mathbf{1 0 7}\end{array}$ & $\mathbf{p}$ \\
\hline Recidiva global (\%) & 14 & 11,2 & 0,24 \\
Recidiva locorregional (\%) & 2 & 2,8 & 0,24 \\
Fallecidos por cáncer (\%) & 12 & 9,3 & 0,26 \\
Quimioterapia adyuvante (\%) & 26 & 34,5 & 0,54 \\
Fallecidos según TNM & & & \\
Estadío I & 0 & 0 & \\
II & 3 & 3 & $0,017 *$ \\
III & 3 & 7 & 0,22 \\
\hline
\end{tabular}

Al comparar estadíos II y III respecto del estadío I en ambos grupos.

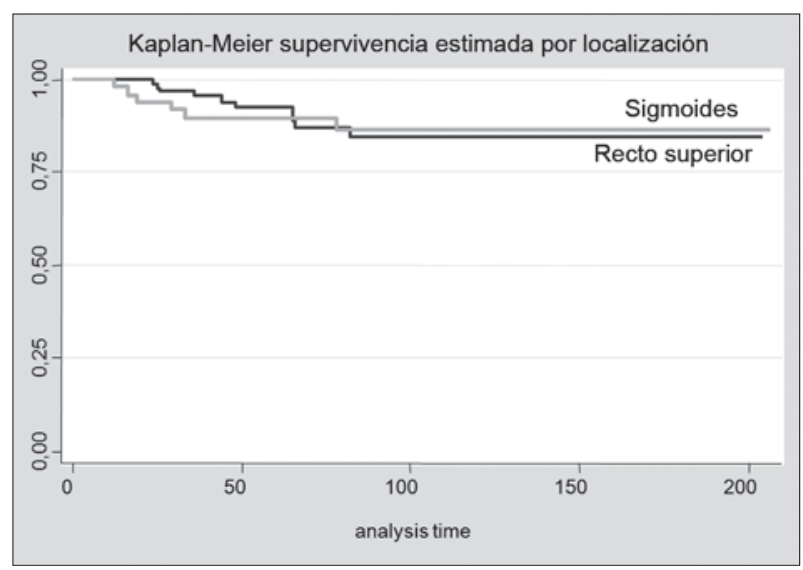

Figura 1. Sobrevida según localización.

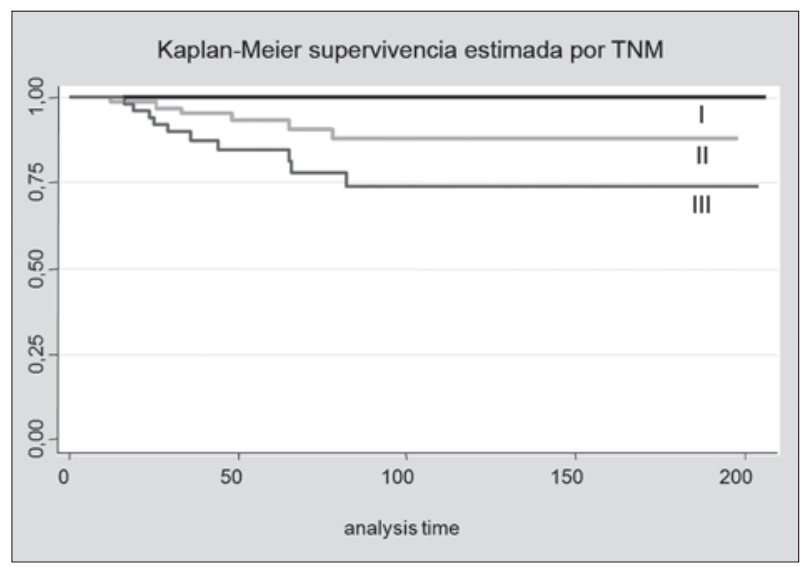

Figura 2. Sobrevida según TNM. cientes en estadío III de RS sometidos a quimioterapia que en los casos en estadío III de CS $(p=0,01)$. No hubo suspensiones ni complicaciones serias asociadas a la quimioterapia.

El seguimiento promedio de los pacientes con un tumor de RS fue de 88,62 meses (extremos 12-206), y 71,67 en los casos de CS (extremos 13-204) $(p=0,05)$. Durante este período fallecen por la enfermedad 6 pacientes $(12 \%)$ del grupo RS, 1 por recidiva locorregional y 5 por recidiva a distancia, con un rango entre 12 y 78 meses. En el grupo CS fallecen 10 pacientes $(9,3 \%), 3(2,8 \%)$ con recidiva locorregional y 7 con recidiva a distancia entre 24 y 82 meses de seguimiento $(\mathrm{p}=$ $0,24)$ (Tabla 2). En el grupo CS 4 pacientes fallecen luego de 5 años de seguimiento. La sobrevida actuarial global a 10 años para ambos grupos fue de $85 \%$ (Figuras 1 y 2 ).

\section{Discusión}

En este estudio comparativo, en forma global, la cohorte de pacientes portadores de un cáncer de RS es similar al grupo de pacientes con un tumor de CS. La distribución por edad, género, incidencia de condiciones comórbidas, albúmina preoperatoria y CEA preoperatorio no muestran diferencias estadísticamente significativas. Sin embargo, el grupo CS se intervino con una anemia preoperatoria de mayor intensidad que el grupo RS, lo que podría traducir diferencias en la oportunidad del tratamiento y, por tanto, del 
tiempo de evolución de una enfermedad que se caracteriza por una gran etapa preclínica ${ }^{10}$. Del mismo modo, no hubo diferencias entre ambos grupos respecto de algunas variables histopatológicas como el grado de diferenciación tumoral, el tamaño de la lesión y, particularmente, la recolección de linfonodos en la pieza operatoria es prácticamente la misma. Esta última variable se ha considerado de gran relevancia en los últimos años como indicador de la calidad y radicalidad de la cirugía y tendría un valor independiente como factor pronóstico. Así, algunas series han demostrado que pacientes con tumores de colon en estadío II tienen un detrimento en la sobrevida cuando el número de linfonodos examinados es inferior a 12 , lo que reflejaría un vaciamiento linfático incompleto $\mathrm{y}$, por tanto, serían lesiones sub-etapifica-das ${ }^{11,12}$.

Si consideramos en forma global la distribución en cuanto a la etapificación de acuerdo al TNM, no habría diferencias estadísticamente significativas entre ambos grupos. Sin embargo, un análisis más detallado revela que hubo una mayor proporción de tumores T4 en el grupo CS, lo que puede influir en los resultados de recidiva local y sobrevida alejada. Aunque en el análisis global no aparecen diferencias significativas en cuanto a la proporción de pacientes que fueron sometidos a quimioterapia adyuvante entre ambos grupos, hubo más pacientes en estadío III tratados en el grupo RS. Esta diferencia no puede ser explicada por la distribución en cuanto a edad o enfermedades asociadas en estos subgrupos, factores relevantes en el acceso a las terapias adyuvantes ${ }^{13}$. El impacto clínico de esta variación no puede ser medida en un estudio de estas características y se requiere de un estudio prospectivo con un gran número de pacientes para lograr demostrar alguna diferencia entre ambos grupos. A nuestro saber, no existe un estudio de esta naturaleza disponible a la fecha.

En este estudio no existe diferencias en cuanto a la recidiva global, la recidiva local ni la recidiva a distancia entre los pacientes intervenidos por un cáncer de CS respecto del grupo con un tumor de RS y la sobrevida actuarial a 10 años es idéntica. En otra serie nacional que compara 60 pacientes de $\mathrm{CS}$ con 59 de RS, aunque la recidiva local fue simi$\operatorname{lar}(\mathrm{p}=0,91)$, hubo diferencias estadísticamente significativas en cuanto a la recidiva a distancia $(90,2 \%$ de sobrevida libre de recurrencia a distancia en CS y $52,4 \%$ en $\mathrm{RS}(\mathrm{p}=0,003)$. Curiosamente, la sobrevida global fue similar en ambas localizaciones $(64,4 \% \text { y } 63,3 \% \text {, respectivamente; } p=0,58)^{14}$. En un estudio de la Cleveland Clinic se comparó los resultados del tratamiento quirúrgico de 229 pacientes con un tumor del tercio superior del recto, en los cuales no se efectuó una resección total del mesorrecto, con 225 pacientes intervenidos por un $\mathrm{CS}$, sin encontrar diferencias en las tasas de recidiva local, metástasis a distancia y recidiva global ${ }^{15}$. Este estudio concluye que los tumores de RS deben tratarse con la misma técnica que las lesiones de CS, lo que es compartido por otros autores ${ }^{16,17}$.

Como conclusión, la distribución por estadíos, el tratamiento quirúrgico estándar y la sobrevida global es similar en los tumores de RS y CS. Los resultados del tratamiento quirúrgico de los cánceres del tercio superior del recto en esta serie apoyarían la conducta de realizar una resección radical con sección a $5 \mathrm{~cm}$ por debajo del nivel del tumor, lo que se ha denominado resección parcial del mesorrecto $^{18}$. Esto no es un problema semántico, considerando la mayor morbilidad asociada con la resección total del mesorrecto que obliga a una desfuncionalización de rutina, según el propio autor del concepto ${ }^{5}$. Como un corolario de lo anterior, no estaría indicado el uso de radioquimioterapia preoperatoria en los tumores de RS, terapia que debe reservarse para las lesiones rectales más bajas ( $\leq 10 \mathrm{~cm}$ del margen anal), cuyo pronóstico es definitivamente más deletéreo ${ }^{15}$. Desafortunadamente, tanto las series nacionales como extranjeras con radioquimioterapia neoadyuvante, incluyen pacientes con tumores del tercio superior de recto, lo que introduce un factor de confusión en los resultados finales ${ }^{2,3,10,19}$. La estrategia de tratar los tumores de RS en forma similar a los de CS estaría justificada de acuerdo con nuestros resultados, evitando técnicas quirúrgicas de mayor complejidad y morbilidad así como tratamientos con radiación que son costosos, tienen serios efectos adversos y alteran la calidad de vida de los pacientes.

\section{Referencias}

1. Li M, Li JY, Zhao AL, Gu J. Colorectal cancer or colon and rectal cancer? Clinicopathological comparison between colonic and rectal carcinomas. Oncology 2007; 73: 52-57.

2. Glynne-Jones R, Harrison M. Locally advanced rectal cancer: What is the evidence for induction chemoradiation? The Oncologist 2007; 12: 1309-1318.

3. Guillem JG, Chessin DB, Cohen AM, Shia J, Mazumdar $\mathrm{M}$, Enker $\mathrm{W}$ et al. Long-term oncologic outcome following preoperative combined modality therapy and total mesorectal excision of locally advanced rectal cancer. Ann Surg 2005; 241: 829-838.

4. Nelson H, Petrelli N, Carlin A, Couture J, Fleshman J, Guillem J, et al. Guidelines 2000 for colon and rectal cancer surgery. J Natl Cancer Inst 2001; 93: 583-596.

5. Heald RJ, Husband EM, Ryall RD. The mesorectum in rectal surgery- the clue to pelvic recurrence? Br J Surg 
1982; 69: 613-616.

6. Otchy D, Hyman NH, Simmang C, Anthony T, Buie WD, Cataldo P, et al. Practice parameters for colon cancer. Dis Colon Rectum 2004; 47: 1269-1284.

7. Bannura G, Cumsille MA, Contreras J, Melo C, Barrera A, Reinero M y cols. Factores pronósticos del cáncer colorrectal: análisis multivariado de 224 pacientes. Rev Méd Chile 2001; 129: 237-246.

8. Bannura G, Cumsille MA, Contreras J, Barrera A, Soto D, Melo C. CEA preoperatorio como factor pronóstico independiente en cáncer de colon y recto. Rev Méd Chile 2004; 132: 691-700.

9. Fleming ID, Cooper JS, Henson DE, Hutter RVP, Kennedy BJ, Murphy GP et al. Editors. American Joint Committee on Cancer. Cancer manual staging. $5^{\text {th }}$ edition, 1997.

10. Weissenberger C, Geissler M, Otto F, Barke A, Henne $\mathrm{K}$, Oplehn $\mathrm{G}$ et al. Anemia and long-term outcome in adjuvant and neoadjuvant radiochemotherapy of stage II and III rectal adenocarcinoma: The Freiburg experience (1989-2002). World J Gastroenterol 2006; 12: $1849-1858$.

11. Chen SL, Bilchick AJ. More extensive nodal dissection improves survival for stages I to III of colon cancer. A population-based study. Ann Surg 2006; 244: $602-$ 610.

12. Swanson RS, Compton CC, Stewart AK, Bland KI. The prognosis of T3N0 colon cancer is dependent on the number of lymph nodes examined. Ann Surg Oncol 2003; 10: 65-71.

13. Jensen SA, Vilmar A, Sorensen JB. Adjuvant chemotherapy in elderly patients ( $>$ or $=75 \mathrm{yr}$ ) completely resected for colon cancer stage III compared to younger patients: toxicity and prognosis. Med Oncol 2006; 23: 521-531.

14. Zárate A, Pinedo G, Loureiro C, Viviani P, Zúñiga A. Comparación de los resultados quirúrgicos y sobrevida entre pacientes con cáncer del tercio superior del recto y colon sigmoides. Rev Chil Cir 2007 (Supl); 59: 89.

15. Lopez-Kostner F, Lavery IC, Hool GR, Rybicki LA, Fazio VW. Total mesorectal excision is not necessary for cancers of the upper rectum. Surgery 1998; 124 : 612-617.

16. Hainsworth PJ, Egan MJ, Cunliffe WJ. Evaluation of a policy of total mesorectal excision for rectal and rectosigmoid cancers. Br J Surg 1997; 84: 652-656.

17. Leong A. Selective total mesorectal excision for rectal cancer. Dis Colon Rectum 2000; 43: 1237-1240.

18. Law WL, Chu KW. Anterior resection for rectal cancer with mesorectal excision. A prospective evaluation of 622 patients. Ann Surg 2004; 240: 260-268.

19. Sauer R, Becker H, Hohenberger W, Rödel C, Wittekind $\mathrm{C}$, Fietkau R et al. Preoperative versus postoperative chemoradiotherapy for rectal cancer. N England J Med 2004; 351: 1731-1740. 